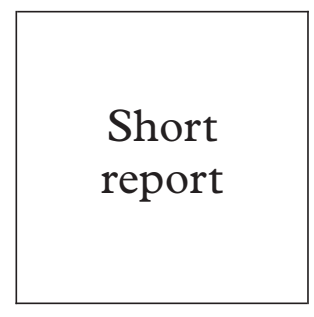

Short report

Department of

Genito-urinary

Medicine, Sunnybank

Wing, Leeds General

Infirmary, Great

George Street, Leeds

LS1 3EX, UK

P P Walker

C Brown

M T Reynolds

PHLS Mycology

Reference Laboratory,

University of Leeds

$\mathrm{H}$ R Ashbee

E G V Evans

Correspondence to: Dr M T Reynolds

m.reynolds@btinternet.com

Accepted for publication 18 July 2000

\title{
Vaginal yeasts in the era of "over the counter" antifungals
}

\author{
P P Walker, M T Reynolds, H R Ashbee, C Brown, E G V Evans
}

Objective: To establish whether there has been any rise in the prevalence of non-albicans Candida species isolated from vaginal swabs since the introduction of "over the counter" antifungal treatments.

Method: A retrospective review looking at all positive vaginal yeast isolates collected from women attending one genitourinary medicine clinic during the 6 year period from 1993 to 1998 inclusive. All positive vaginal yeast isolates were included, regardless of whether or not the patients were symptomatic. Isolates from HIV positive women were excluded from the analysis. Result: No increase in non-albicans vaginal yeast isolates was shown during the period studied. The proportion of non-albicans yeasts remained constant at approximately $5 \%$ of the total yeasts isolated. The most common non-albicans yeast isolated was $C$ glabrata.

Conclusion: There is no evidence from this study to suggest that the increasing use of "over the counter" antifungal treatment has selected for atypical, possibly inherently azole resistant, strains of vaginal yeasts in HIV seronegative women.

(Sex Transm Inf 2000;76:437-438)

Keywords: vulvovaginal candidiasis; non-albicans species; antifungal drug resistance

\section{Introduction}

It is estimated that $75 \%$ of women will experience at least one episode of vulvovaginal candidiasis in their lifetime and $40-50 \%$ will experience two or more episodes. ${ }^{1}$ Candida albicans accounts for the majority of cases of vulvovaginal candidiasis, but up to $10 \%$ are due to nonalbicans yeast species. ${ }^{2} 3$

"Over the counter" treatments for vulvovaginal candidiasis have now been licensed for a number of years. Clotrimazole pessaries were first made available for purchase by the general public in 1992 and a fluconazole single dose oral tablet ("Diflucan One", Pfizer) in 1995. Unit pack sales of Diflucan One almost doubled from 367115 in 1996 to 661888 in 1998 (Pfizer Consumer Healthcare, personal communication). Undoubtedly the availability of "over the counter" antifungals is convenient for large numbers of women. However, there is evidence that many women have difficulty in diagnosing candidiasis correctly ${ }^{4}$ and it could be that increasing or inappropriate use of "over the counter" preparations might select for atypical strains of vaginal yeasts.

The aim of this study is to look at yeast cultures performed on vaginal swabs taken from women attending a busy genitourinary medicine clinic to see if there has been any rise in the prevalence of non-albicans Candida species found since the introduction of "over the counter" antifungals.

\section{Methods}

This is a retrospective review performed at a busy city centre genitourinary medicine clinic. All positive vaginal yeast isolates collected from women attending between 1993 and 1998 inclusive were included, regardless of whether or not the patients were symptomatic. Any isolates from HIV seropositive women were excluded. Any vaginal yeast species isolated from an individual patient was counted only once, when first isolated from that patient.

Yeasts isolated from patients were identified using standard mycological techniques. ${ }^{5}$ The purity of cultures was confirmed and each isolate was tested for the ability to produce germ tubes. Germ tube positive isolates were identified as $C$ albicans. Germ tube negative isolates were identified using commercially available kits, including Fongiscreen (Sanofi Pasteur), API 32C (Bio Merieux), and Auxocolor (Sanofi Pasteur). If fewer than 10 colonies were isolated from a patient and they were germ tube negative, they were not fully identified, but designated as "non-albicans" yeasts.

\section{Results}

No increase in non-albicans vaginal yeast isolates was shown during the period studied. The results are detailed in table 1 . Of the women sampled, between $25-27 \%$ were culture positive for yeasts over the 6 years studied. The non-albicans yeast species found are

Table 1 Vaginal yeast isolates, 1993-8

\begin{tabular}{|c|c|c|c|c|c|c|}
\hline & 1993 & 1994 & 1995 & 1996 & 1997 & 1998 \\
\hline Total vaginal fungal cultures performed & 3950 & 4400 & 4484 & 5265 & 5427 & 5646 \\
\hline Total $C$ albicans isolated & 946 & 1069 & 1126 & 1240 & 1381 & 1456 \\
\hline$C$ albicans $<10 \mathrm{CFU}^{\star}$ & 261 & 255 & 273 & 275 & 330 & 385 \\
\hline Total non-albicans Candida isolated (including mixed cultures) & 52 & 61 & 53 & 56 & 61 & 65 \\
\hline Non-albicans $<10 \mathrm{CFU}^{\star}$ & 19 & 24 & 13 & 22 & 28 & 21 \\
\hline Percentage of positive vaginal fungal cultures & $25 \%$ & $26 \%$ & $26 \%$ & $27 \%$ & $27 \%$ & $27 \%$ \\
\hline Percentage of yeast isolates that were non-albicans & $5.2 \%$ & $5.4 \%$ & $4.5 \%$ & $4.3 \%$ & $4.2 \%$ & $4.3 \%$ \\
\hline
\end{tabular}

$\star<10 \mathrm{CFU}=$ fewer than 10 colonies isolated. 
Table 2 Non-albicans yeast species isolated, 1993-8

\begin{tabular}{|c|c|c|c|c|c|c|}
\hline & 1993 & 1994 & 1995 & 1996 & 1997 & 1998 \\
\hline$C$ glabrata & 17 & 24 & 24 & 15 & 20 & 24 \\
\hline$C$ parapsilosis & 3 & 2 & 2 & 5 & 5 & 3 \\
\hline$S$ cerevisiae & 4 & 3 & 3 & 4 & 1 & 6 \\
\hline C tropicalis & 1 & 3 & 2 & 3 & 2 & 1 \\
\hline C krusei & 1 & & & 2 & & 2 \\
\hline Clusitaniae & 1 & & 1 & 1 & 2 & 3 \\
\hline$C$ famata & 1 & & & & 1 & \\
\hline C inconspicua & & & 1 & & 1 & \\
\hline C kefyr & & & & 2 & & \\
\hline Trichosporon & 1 & 1 & 2 & & & \\
\hline $\begin{array}{l}\text { Germ tube negative yeasts, } \\
\text { not fully identified }{ }^{\star}\end{array}$ & 19 & 24 & 13 & 22 & 28 & 21 \\
\hline Mixed species & 4 & 4 & 5 & 2 & 1 & 5 \\
\hline Total & 52 & 61 & 53 & 56 & 61 & 65 \\
\hline
\end{tabular}

^If fewer than 10 colonies were isolated from a patient and they were germ tube negative, they were not fully identified. candidiasis, ${ }^{11}$ but so far it has not been demonstrated except in a very highly selected population of women, who were HIV seropositive. ${ }^{12}$

How many of the women sampled in this study had previously treated themselves with antifungal preparations is unknown. Nevertheless, the study demonstrates that, at our centre, there has been no increase in the isolation of non-albicans vaginal yeasts over a 6 year period during which time the use of "over the counter" antifungals has increased enormously. Thus, so far, we have found no evidence that the increasing use of these antifungals has selected for atypical, possibly inherently azole resistant, strains of vaginal yeasts in HIV seronegative women.

shown in table 2. The proportion of nonalbicans yeasts remained constant at approximately $5 \%$ of the total yeasts isolated. The most common non-albicans species isolated was $C$ glabrata, which made up $47-71 \%$ of the non-albicans species that were fully identified, - that is, more than 10 colonies isolated per swab.

\section{Discussion}

Fluconazole resistance is well recognised in HIV seropositive individuals with oropharyngeal and oesophageal candidiasis, and has been shown to be related to previous fluconazole exposure. ${ }^{6}$ In contrast, vulvovaginitis due to azole resistant $C$ albicans is exceptionally rare in HIV seronegative women. ${ }^{7}$ For example, in a group of women with recurrent vulvovaginal candidiasis caused by $C$ albicans, no azole resistance was demonstrated by successive isolates despite at least 6 months' maintenance therapy with either oral ketoconazole or fluconazole. ${ }^{8}$

The use of azole antifungals, particularly fluconazole, may also lead to a pathogen shift with increased incidence of the inherently resistant Candida species such as $C$ glabrata and $C \mathrm{kru}-$ sei. ${ }^{9}$ Such a pathogen shift in Candida species has been observed in bloodstream infections. ${ }^{10}$ It has been suggested for a number of years that this may also have occurred in vulvovaginal
Contributors: PPW collected and analysed the data and wrote the first draft of the manuscript; MTR initiated the project, helped with collection and analysis of the data and revised the manuscript; CB and HRA helped with data collection; HRA and EGVE supervised the laboratory testing, provided technical advice, and revised the manuscript.

1 Sobel JD. Epidemiology and pathogenesis of recurrent vulvovaginal candidiasis. Am f Obstet Gynecol 1985;152:92435.

2 Denning DW, Baily GG, Hood SV. Azole resistance in candida. Eur f Clin Microbiol Infect Dis 1997;16:261-80.

3 British Society for Medical Mycology Subcommittee on Diagnostic Mycology. Management of genital candidiasis. BMF 1995;310:1241-44.

4 Ferris DG, Dekle C, Litaker MS. Women's use of over-thecounter anti-fungal medications for gynecologic symptoms. counter anti-fungal medications for

5 Evans EGV, Richardson MD, eds. Medical mycology-a Evans EGV, Richardson MD, eds. Medical mycology-a
practical approach. Oxford: Oxford University Press, 1989.

6 Maenza JR, Keruly JC, Moore RD, et al. Risk factors for fluconazole-resistant candidiasis in human immunodeficiency virus-infected patients. F Infect Dis 1996;173: $219-25$

7 Sobel JD, Vazquez JA. Symptomatic vulvo-vaginitis due to fluconazole-resistant Candida albicans in a female who was Infect Dis 1996;22:726-7.

8 Lynch ME, Sobel JD, Fidel PL Jr. Role of anti-fungal drug resistance in the pathogenesis of recurrent vulvovaginal candidiasis. F Med Vet Mycol 1996;34:337-9.

9 Lynch ME, Sobel JD. Comparative in vitro activity of antimycotic agents against pathogenic vaginal yeast isolates. $\mathcal{F}$ Med Vet Mycol 1994:32:267-4.

10 Price MF, LaRocco MT, Gentry LO. Fluconazole susceptibilities of Candida species and distribution of species recovered from blood cultures over a 5-year period. Antimi-

11 Sobel JD. Vaginal infections in adult women. Med Clin North Am 1990;74:1573-601.

12 Spinillo A, Capuzzo E, Gulminetti R, et al. Prevalence of and risk factors for fungal vaginitis caused by non-albicans species. Am f Obstet Gynecol 1997;176:138-41. not infected with human immunodeficiency virus. Clin crob Agents Chemother 1994;38:1422-4. 